

\section{Sumário}

I. Dossiê Especial: History of International Law ...................................1

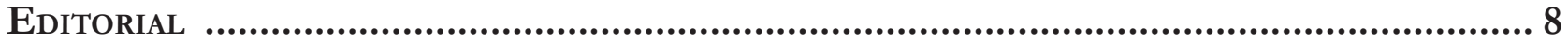

What does it mean to apply history in international law studies? ....................................................... 8

Arthur Roberto Capella Giannattasio

SuR LA NATURE DU Droit ISLAMIQUe............................................................................14

Hocine Benkheira

Islamic Shari’a Law, History and Modernity: Some Reflections .................................25 Suleiman A. Mourad

The (Un)practical Secularization Process: International Law and Religion as So-

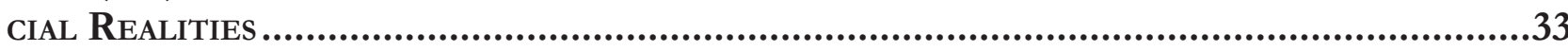

Douglas de Castro

BRAZILIAN LITERATURE ON INTERNATIONAL LAW DURING THE EMPIRE REGIME. OR THE DIFFUSION OF INTERNATIONAL LAW IN THE PERIPHERIES THROUGH APPROPRIATION AND ADAPTATION.

Airton Ribeiro da Silva Júnior

Natural, POSitivo, romano E Universal? INVESTigaÇão SObre O Direito das GENTES EM

Tomás de Aquino

Rafael Zelesco Barretto

II. Artigos sobre outros temas

VINCULAÇÃO DOS DIREITOS ECONÔMICOS, SOCIAIS E CULTURAIS: UMA DISCUSSÃO DO DESENVOLVImento humano com base no conceito de Amartya Sen sobre o mínimo existencial.....99

Natalia Mascarenhas Simões Bentes e Yasmim Salgado Santa Brígida

A NOVA LeI de MigraÇão E A PROTEÇão CONFERIDA AO APÁtrida: ALINHAMENTO BRASILEIRO AO PADRÃO INTERNACIONAL DE DIREITOS HUMANOS

Pedro Henrique de Faria Barbosa e Sylvio Loreto

E se o Supremo Tribunal Federal (STF) restabelecer a vigência da Convenção n. 158 
da Organização Internacional do Trabalho (OIT) na ordem Jurídica brasileira? SoBRE UMA POSSÍVEL REVIRAVOLTA, PELA VIA DO DIREITO INTERNACIONAL, DAS LEIS TRABALHISTAS BRASILEIRAS 138

Daniel Damasio Borges

JULGADOS DA CORTE INTERAMERICANA SOBRE CASOS BRASILEIROS E POLÍTICAS PÚBLICAS: REFLEXÕES ACERCA DE POSSÍVEIS INFLUIÇÕES 165

Rafael Osvaldo Machado Moura

CREATING BRIDGES BETWEEN INTERNATIONAL RELATIONS THEORY AND INTERNATIONAL HUMAN RIGHTS LAW: CONSTRUCTIVISM AND THE ROLE OF BRAZIL IN THE INTER-AMERICAN SYSTEM OF HUMAN RIGHTS 179

Ismael Francisco de Souza, Luciana Rocha Leme e Erick da Luz Scherf

Justiça de transição na Argentina e o Sistema Interamericano de Direitos Humanos: uMa ANÁlise do CASo Luis Muiña (“REgRa 2x1”) 199

Emilio Peluso Neder Meyer e Jessica Holl

A legalidade e legitimidade da INTERVEnÇão humanitÁria: UMA MEDIDA AINDA NECESSÁRIa.219 Natália Caye Batalha Boeira

O Acordo de Escazú E o ACESSo À INFORMaÇão AMbiental no Brasil. 252 Érica Bezerra Queiroz Ribeiro e Bruno Amaral Machado

Dos POVOS NATIVOS AO SURGIMENTO DOS MOVIMENTOS SOCIAIS: INFLUÊNCIAS DOS DISCURSOS JURÍDICOS, RELIGIOSOS E MÉDICOS PARA A CONSTRUÇÃO DO CONCEITO DE HOMOSSEXUALIDADE NO BRASIL .267 Bruno Rafael Silva Nogueira Barbosa e Robson Antão de Medeiros

Aspectos Jurídicos da PARTicipaÇão dA UNião Europeia NA OMC: COMPREENDENdo SUTILEZAS DE UM DELICADO ENLACE. 291

Camilla Capucio

Path to judicial activism? The use of "Relevant rules of international law" by the WTO Appellate Body

Mariana Clara de Andrade

LEVEZA E PESO NA MEDIAÇÃO COMERCIAL INTERNACIONAL: O CONTEÚDO JURÍDICO DO ACORDO CORPORATIVO MEDIADO E SUA INCORPORAÇÃo PELO DiREITO BRASILEIRO .324 Henrique Lenon Farias Guedes 
JURISDIÇÃO INTERNACIONAL E AS DIFICULDADES DE EXECUÇÃO DE SENTENÇAS INTERNACIONAIS NO BRASIL

Nevitton Vieira Souza

O DEVER DE COOPERAÇÃo NOS CONTRATOS DE VENDA INTERNACIONAL DE MERCADORIAS: PRESSUPOSTOS TEÓRICOS E REPERCUSSÕES PRÁTICAS DA CLÁUSULA GERAL DA BOA-FÉ OBJETIVA PARA A APLICAÇÃo DA CISG .358

Angelo Gamba Prata de Carvalho

A DiMENSÃo JURÍdiCA DO IMPERIALISMO NA (DES)ORDEM GLOBAL CAPITALISTA: UMA ANÁLISE COM BASE NA CRÍTICA MARXISTA AO DIREITO INTERNACIONAL E ÀS RELAÇÕES POLÍTICO-ECONÔMICAS DE DOMINAÇÃO E DEPENDÊNCIA.

Thomaz Delgado De David, Maria Beatriz Oliveira da Silva e Rosane Beatris Mariano da Rocha Barcellos Terra

A participaÇão de Brasil e Estados Unidos na formulação das regras multilaterais do

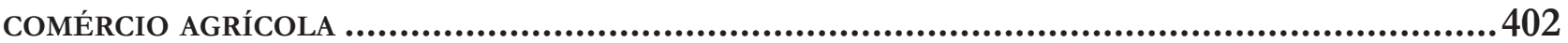

Vera Thorstensen, Vivian Daniele Rocha Gabriel e Alebe Linhares Mesquita

A galáxia lex e a construÇão de um Sistema jurídico transnacional ........................ 441

Eugênia Cristina Nilsen Ribeiro Barza e Jéssyka Maria Nunes Galvão

Has the Ability of Truth Commissions to Recommend Amnesty Been Effective in Enhancing Perpetrator Cooperation? 453 Jeremy Sarkin

A CONCEPTUAL PAPER ON THE POLICY-FRAMEWORK THAT MIRRORS THE DYNAMIC LINK BETWEEN Human Security, Social Protection and Safety Nets, and Food and Nutritional Security: The Case of the "Gulayan sa Paaralan Program", the Philippines.... 478 Renato Lagapa Base

INCENTIVISING SMALLHOLDER FARMER LIVELIHOODS AND CONSTRUCTING FOOD SECURITY THROUGH HOME-GROWN SCHOOL FEEDING: EVIDENCE FROM NORTHERN GHANA .491

Clement Mensah

Policy COHERENCE In THE IMPLEMENTATION OF THE 2030 AgENDA FOR SUSTAINABLE DEVELOpment: the Brazilian School Feeding Programme Case Study .506 Mariana Werlang Girardi 


\title{
Sur la nature du Droit Islamique*
}

\section{Sobre a Natureza do Direito islâmico}

\author{
On the Nature of Islamic Law
}

Hocine Benkheira**

\section{RÉSUMÉ}

L'expression «droit musulmans » ou «droit islamique » pourrait laisser croire à l'existence d'un modèle juridique universel. Dans notre contribution nous cherchons à souligner certaines spécificités du système institutionnel de l'islam, comme par exemple le fait que le rôle central est joué non par le juge, mais par le juriste ou mufti. C'est ce fait qui empêche la subordination du système juridique au système politique. Il en est autrement de nos jours alors que la plupart des pays musulmans ont adopté un modèle d'inspiration occidental.

Mots-clés: droit islamique; Moyen Orient ; Juriste ; Interprétation ; Droit révélé

\section{Abstract}

The term «Muslim law» or «Islamic law» might suggest the existence of a universal legal model. In our contribution we seek to highlight some specificities of the institutional system of Islam, such as the fact that the central role is played not by the judge, but by the jurist or mufti. It is this fact that prevents the subordination of the legal system to the political system. It is different nowadays when most Muslim countries have adopted a Westerninspired model.

Keywords : islamic Law - Middle-East - jurists - interpretation - revealed Law

\section{Un PROBLĖME de VOCABULAIRE.}

Mon exposé d'aujourd'hui porte sur du droit, non pas sur une société ou sur une théorie. En français, comme dans d'autres langues européennes, on distingue droit et loi (en allemand, recht/ gesetv). ${ }^{1}$ Existe-t-il des mots similaires en arabe ? Il faut probablement distinguer selon que l'on parle

\footnotetext{
* Auteur invité / Guest author / Autor convidado.

1 A titre comparatif, voir «Jus» de Georges Dumézil, Idées romaines, 2e édition, Gallimard, 1980 [1969], 31-45, ainsi qu’Emile Benvéniste, Le vocabulaire des institutions indo-européennes, Minuit. 
de l'arabe, avant l'apparition de l'islâm, ou de l'arabe, qui a été profondément marqué par l'islâm. L'arabe dispose d'une série de termes : sunna, shar sharita, fiqh, qânûn. Aucun n'équivaut à droit, sauf fiqh, mais qui a une autre signification. Pour parler des «droits » de la personne, on utilise un mot différent : haqq, pl. buqûq. Ce terme a d'autres sens : notamment il peut signifier "vrai » (opposé à bâtil, « faux »), de là haqîqa, "vérité », mais aussi « réalité »; il peut même désigner Dieu (nom divin). C'est seulement à l'issue d'un long processus complexe que fiqh a fini par désigner « le droit islamique ", dans un sens très large. Le mot fiqh peut être traduit par « intelligence » (au sens actif, dynamique, du verbe 'intelliger'), " compréhension », voire même « science » (on rencontre l'expression fiqh al-lugha, " science de la langue »). Il y a eu pendant longtemps une compétition entre figh et 'ilm (science). On désigne le juriste par les mots faqîh ou 'âlim. Mais ce dernier terme peut être appliqué à toute personne savante. Aujourd'hui, sans doute en raison de l'appropriation, dès la période classique, du terme 'ilm par la falsafa, une discipline profane et surtout exogène, c'est ce même terme qui sert à désigner les sciences au sens moderne. ${ }^{2}$ Notez qu'un juriste très savant peut être affublé du titre de 'allâma, "le très savant ». Ainsi sont surnommés, par exemple du côté sunnite Ibn Khaldoun, comme du côté imâmite al-Hillî.

Le droit, dans tous les cas, doit être distingué à la fois des phénomènes sociaux mais aussi du monde des idées. On ne peut envisager une règle de droit comme on envisage une élection présidentielle ou une guerre. Mais une règle de droit, même si elle a un rapport étroit au monde des idées, n'est pas qu'une idée ou un ensemble d'idées. Le droit définit dans toute société l'espace dans lequel évoluent les sujets. C'est pour cela qu'il n'est pas faux de dire que, dans toute société et à toutes les époques, quelle que soit la forme qu'il prend, écrit ou oral, savant ou coutumier, il est le « squelette » de la société. Le droit n'est pas seulement important dans le monde islamique, il l'est également dans le monde occidental moderne. Il y a toutefois de nombreuses différences entre les deux univers, dont, sans doute, la plus importante est la désacralisation qui a atteint le droit occidental moderne. La loi n'est plus sacrée au même niveau où elle l'était dans les mondes anciens et les systèmes juridico-religieux. Il y a d'autres différences

2 Cet usage est déjà attesté chez Ghazzâlî, dans l'Ibyâ'. importantes. En France, on tient pour évidentes des distinctions comme droit canonique/droit civil (au sens de droit romain), droit privé/droit public ou droit naturel/droit positif. En islâm, les distinctions qui sont de mises sont tout autres : on distingue le droit positif (furû) de la théorie légale (usû́), le droit du culte ('ibâdât $)$ du droit des contrats (mu'âmalât) ou la sharîa de la coutume ('urf, 'âda). Il n'y a pas par exemple de droit constitutionnel et les divisions si familières au juriste français (droit des personnes, droit commercial, droit administratif, etc.) sont inconnues.

Nous désignons par «droit islamique » ce que les musulmans appellent habituellement fiqh, un mot étrange qui signifie au départ "intelligence ", " compréhension », sans doute aussi " science », en raison de son rapprochement et de sa confusion avec le mot 'ilm, «science », « savoir ». C'est pour cela qu'à partir d'une certaine époque, on a désigné indifféremment le juriste par les mots faqîh, pluriel fuqahâ', et 'âlim, pluriel 'ulamâ' (d'où le français " ouléma »). Il existe un autre mot que vous connaissez, c'est shari a, que l'on traduit habituellement par « loi islamique ». On peut distinguer fiqh et shar ${ }^{\star} a$, dans la mesure où cette dernière constitue un résultat auquel parvient le docteur de la Loi, le 'âlim, quand il parvient à cerner les contours de la loi islamique et à en préciser le contenu prescriptif (prescriptions positives et négatives), alors que le fiqh c'est l'ensemble des disciplines intellectuelles qui sont mobilisées afin de parvenir à cette fin ainsi que le résultat lui-même.

Aujourd'hui, et notammentdu point de vue occidental, ce qui caractérise avec force le DI, est sa relation à une religion, dont il est un élément constitutif majeur, mais tout de même un simple élément. Autrement dit, intégré dans la structure du système religieux de l'islâm, le DI est pour cette raison assujetti à ce même système religieux. Considéré du point de vue d'aujourd'hui, où l'idée de loi de Dieu ou même l'idée plus générale d'une loi sur laquelle les humains n'auraient pas prise est devenue inadmissible, cette particularité du DI engendre, non seulement de l'incompréhension mais, ce qui est plus surprenant, une forte hostilité. Si nous nous plaçons à la fin de l'Antiquité tardive, l'idée de loi révélée était sans doute aussi répandue que l'est aujourd'hui la conception de la démocratie représentative. ${ }^{3}$ Les conceptions juridico-politiques sont des phénomènes historiques et,

3 Voir Rémi Brague, La loi de Dieu. Histoire d'une alliance philosophique, Gallimard, coll. Folio, 2005. 
par conséquent, aucune n'est ni naturelle ni éternelle. Du reste, on oublie que même dans les démocraties, un partie importante du droit n'a jamais été adoptée par les parlements mais hérité des institutions passées, comme la règle de la non rétroactivité des lois ou celle du consentement des parties dans le contrat.

En relation avec cette particularité, on doit évoquer trois problèmes qui sont distincts. Premièrement, l'opposition entre loi divine et loi humaine n'est pas si tranchée qu'il y parait. Commençons par rappeler que la loi divine est imputée à la divinité comme au principe fondateur, qui échappe à l'influence humaine. On peut à bon droit considérer de ce point de vue que le Dieu, Auteur des lois, n'est qu'une fiction institutionnelle. S'agissant du droit islamique, il est le produit d'une tentative de concilier un message religieux, des traditions juridiques préislamiques et l'usage du raisonnement. Si nous considérons le droit français contemporain, par exemple, il est imputé au Peuple ou à la Nation; dans les deux cas, ces deux entités sont également des fictions institutionnelles, car si personne ne peut prétendre avoir rencontré Dieu, nul non plus n'a vu le Peuple ou la Nation. Il ne s'agit pas cependant de conclure que Dieu et le Peuple (ou la Nation), c'est la même chose : ces fictions sont de nature différente. Il en va de même si nous rapportons le droit occidental contemporain à la Raison, car, ce faisant, nous élevons celle-ci au statut de force cosmique. Il faut indiquer qu'il y a une certaine cohérence de la part des oulémas à rapporter le DI à Dieu, car sur le plan théologique, ils défendent le concept d'un Dieu tout-puissant et interventionniste, qui n'admet aucun autre sujet que lui-même : l'homme n'est un sujet que sous l'autorité du Sujet monumental divin. Sur ce plan, il y a peu d'intérêt à opposer sunnisme et chiisme (sauf peut-être à une certaine époque, sa variante ismaélienne).

Deuxièmement, on ne doit pas confondre le DI avec le droit des pays musulmans contemporains, sauf quelques cas qui méritent d'être examinés de près (Arabie saoudite, Iran, Somalie, Lybie). Dans la plupart des autres pays musulmans, sauf le cas turc, le droit islamique constitue une source plus ou moins importante de la législation en vigueur dans chacun de ces pays. C'est surtout le droit civil, en particulier le droit des personnes, de la famille et des successions, qui reste dépendant du droit islamique. Malgré tout, il est rare que la législation en vigueur soit simplement inspirée du DI. Très souvent, cette législation est pénétrée de concepts d'origine occidentale. Exemples : substitution de la distinction mineur-majeur à impubère-pubère, état-civil, patronyme, etc. On doit observer que même dans le cas turc, l'occidentalisation du droit est demeurée superficielle et n'a touchée que la partie urbaine et éduquée de la société. Les populations turques émigrées en Europe sont ainsi celles qui pratiquent le plus «le mariage arrangé », qui, aux yeux des droits ouesteuropéens est sinon un délit, un abus de pouvoir dans la mesure où le groupe de parenté fait pression sur l'individu (généralement, les filles) afin qu'il donne son accord à une union qu'il refuserait autrement. Le kémalisme a cherché à transformer la société par en haut, en transformant le droit, mais, comme dans les pays socialistes, on a violenté la société, qui a seulement attendu le moment propice pour prendre sa revanche. Par là même, on doit observer que le droit islamique continue d'être vivant, mais de manière qui est assez différente de ce que fut sa situation à l'Age classique (du $\mathrm{III}^{\mathrm{e}} / \mathrm{IX}^{\mathrm{e}}$ au XIII' $/ \mathrm{XIX}^{\mathrm{e}} \mathrm{s}$.).

Troisièmement, la comparaison qui est parfois faite entre le DI et le droit canonique est inexacte, car ce dernier a toujours existé aux côté d'un droit civil, ou de coutumes, ou plus tard une législation impériale ou monarchique. La totalité de la vie sociale n'est pas régie par le droit canonique, qui règlement en priorité la vie des ecclésiastiques et les rapports des laïcs aux prescriptions religieuses. Par exemple, le droit canonique ne se préoccupe ni de droit commercial ni de la succession dynastique. Par ailleurs, le droit canonique est l'œuvre de l'Eglise, qui se conçoit comme une société à part entière, distincte de la société laïque, alors qu'une telle construction fait complètement défaut du côté islamique. Il est vrai que le droit canonique a longtemps régi les mariages et d'autres domaines, laïcisés depuis longtemps. Mais le mariage n'est tombé sous l'autorité du droit canonique que parce qu'il a été défini par la théologie comme un sacrement, donc un acte religieux, comme le blasphème ou le serment. Le DI est seul à régner en terre d'islâm : la dichotomie entre un droit religieux et un droit séculier non seulement absente mais impensable en terre d'islâm - sauf dans la cas particulier du qânûn, qui désigne l'ensemble des règles édictées par les pouvoirs en place, à condition que ces règles n'empiètent pas sur le champ d'intervention du DI (ainsi le qânûn ne peut jamais régir la filiation ou les 
successions). ${ }^{4}$

Aussi l'on peut être tenté d'insister sur la nature religieuse du DI puisque c'est, pense-t-on, son contenu religieux qui le définit le mieux. Ce n'est pas inexact, mais ce faisant on risque de passer à côté de l'essentiel. Pour commencer, on doit remarquer que le DI n'est pas intégralement religieux, si l'on met de côté les matières rangées par les juristes musulmans sous la rubrique des 'ibâdât, terme que l'on peut traduire grossièrement par « règles du culte », le reste a peu à voir en général stricto sensu avec la religion.

Prenons l'exemple du mariage. A la différence du christianisme, l'islâm ne propose aucune théologie du mariage, qui est défini comme un contrat ordinaire, qui suppose l'accord des co-contractants, exactement comme une opération commerciale : les juristes comparent souvent le contrat matrimonial à un acte de vente. Le DI énonce un certain nombre de règles pour qu'un mariage soit valide et entraîne ce faisant les effets attendus (légitimité des enfants qui en sont issus, vocation successorale entre les conjoints et entre ces derniers et leur progéniture, transmission du nom du père, création d'empêchements matrimoniaux, obligation alimentaire...). Il précise également les conditions de rupture du lien matrimonial et les obligations qui en découlent pour chacun des conjoints.

La religion peut intervenir dans ce corpus de règles, mais seulement à la marge, dans des domaines très précis : en fait on peut en indiquer deux. D'abord, la disparité religieuse entre futurs conjoints : un musulman ou une musulmane peuvent-ils épouser un conjoint non musulman ? La réponse à cette question, on le sait, dépend du genre, puisque, si, sous certaines conditions, un homme peut épouser une chrétienne ou une juive (ou appartenant à une religion assimilée), une femme ne le peut jamais. On n'échange pas donc de femmes avec les autres communautés religieuses : on leur prend des femmes, mais on ne leur en donne pas. Mais sans doute cette situation est-elle à rapporter à la règle plus générale de la parité entre conjoints (kafầa). En effet, selon cette règle, si un homme peut épouser une femme

4 Un exemple parmi d'autres, emprunté à la situation contemporaine. Au début des années 1980, quand les autorités algériennes ont cherché à établir un Code de la famille, elles ont consulté le Conseil Supérieur Islamique qui a notamment rejeté l'inscription de toute union matrimoniale à l'état-civil comme condition de validité sous prétexte que cette condition est étrangère au DI. de condition sociale et juridique inférieure à la sienne, une femme ne le doit jamais. ${ }^{5}$ Or dans une situation où l'islâm est majoritaire et prédomine, appartenir à une religion "protégée », c'est être un inférieur. Donc la disparité religieuse serait moins une conséquence de l'intervention de la religion dans le contenu normatif que la conséquence d'une conception hiérarchique de la société.

Une autre règle importante du mariage est concernée par la disparité religieuse, c'est la tutelle matrimoniale. Dans ce cas l'appartenance religieuse communautaire est en compétition avec les liens du sang : selon le DI, en cas de disparité religieuse entre un homme et sa fille (ou son fils mineur), il ne peut exercer la fonction de tuteur matrimonial à son égard, qu'il ait été musulman ou non. L'appartenance religieuse prévaut également en matière successorale sur les liens du sang puisque la disparité religieuse est un obstacle à la vocation successorale. Pourtant, on ne peut pas en déduire que c'est une règle générale. Il y a un domaine en particulier, où les liens du sang prévalent explicitement sur l'appartenance confessionnelle : c'est la nafaqa, l'entretien de l'épouse et de tous ceux qui dépendent du sujet (enfants, proches parents, esclaves).

Dans la mesure où la société est organisée sur un mode hiérarchique stricte, l'appartenance religieuse intervient comme un des principaux critères de hiérarchisation. C'est en fait moins de religion au sens habituel dans le monde occidental moderne qu'il faut parler que d'un mode d'être dans le monde. Le quiproquo au sujet du DI, quand on l'aborde d'un point de vue occidental, est qu'il est étranger au dispositif qui a fini par prévaloir en Europe de l'Ouest, à savoir l'émergence d'un Etat, maitre des lois et seul titulaire de la souveraineté. ${ }^{6}$ Les parlements d'aujourd'hui comme la démocratie sont les formes d'épanouissement de ce dispositif. La principale singularité du modèle occidental est que l'Etat (qui possède, en droit international, mais pas seulement, la

5 Il y a tout de même une entorse théorique à cette règle fondamentale : on admet qu'une femme de condition libre épouse un esclave ou affranchi. Mais on est en droit de se demander si cette possibilité n'est pas un « reste » des origines égalitaristes du DI, les anciens Arabes ayant été toujours hostiles à ces unions mixtes.

6 Le maitre-livre dans ce domaine est encore le Léviathan de Thomas Hobbes (XVIIe), qui est la principale source d'inspiration du Contrat social de J.J. Rousseau, comme l'a bien montré F. Derathé (JJ Rousseau et la science politique de son temps, Vrin). Parmi plusieurs manuels d'histoire du droit, on peut citer Jean-Marie Carbasse, Manuel d'introduction historique au droit, PUF, 2009 (troisième édition refondue), voir notamment le chapitre 15 («Le roi législateur »). 
personnalité morale, donc la capacité d'agit comme un sujet de droit, autrement on ne pourrait parler de souveraineté à son sujet) devient le principal législateur. Même si en même temps le contrat est source du droit, surtout dans le domaine économique, il l'est sous l'égide et dans le cadre défini par l'Etat. Sans l'ordre juridique instauré ou préservé par l'Etat, aucun engagement n'aurait de valeur, sauf peut-être entre partenaires de même force. On insiste beaucoup sur l'aspect démocratique ; mais, tout compte fait, la différence entre la monarchie absolutiste et la démocratie parlementaire est un détail technique. Dans un cas c'est le monarque qui édicte les lois, dans l'autre, c'est le Chef de l'Etat après que le parlement les ait adoptées. La monarchie absolutiste ne signifie pas que les lois n'étaient pas pensées ni discutées avec profondeur et pénétration. L'absolutisme n'est pas le règne de l'arbitraire, sauf dans une image d'Epinal. C'est pour cela que la formule, d'origine allemande « Etat de droit» (staatrecht, rule of law en anglais) est ambiguë : c'est comme si avant « l'Etat de droit», régnait «l'Etat de la force».

On peut invoquer un autre aspect du DI en faveur de sa nature religieuse : le DI n'a pas une validité universelle. Autrement dit, non seulement il ne s'applique pas à la terre entière, mais il ne s'applique même pas aux membres des religions " protégées » qui vivent en terre d'islâm, qui demeurent tant qu'ils le souhaitent sous la juridiction de leurs propres communautés, sauf dans les cas où ils entrent en conflit avec un(e) musulman(e) ou s'ils souhaitent eux-mêmes, librement, se soumettre au DI (par exemple, pour échapper au pouvoir de leur propre communauté). Le fait qu'en cas de litige entre un musulman et un "protégé ", c'est le DI qui prévaut et s'applique est une conséquence de la hiérarchisation de la société, que nous évoquions précédemment. C'est pour cela que l'on peut définir le DI comme « non territorial » stricto sensu, car il se rattache aux personnes qui adhèrent à l'islâm, non à celles qui vivent en terre musulmane. Expliquons cet aspect. Dans le droit occidental contemporain, on ne peut se prévaloir de son origine nationale pour refuser de se voir appliqué le droit du pays où l'on réside, sauf conditions prévues par la Loi elle-même (accords entre Etats, notamment). Un musulman, quel que soit sa nationalité, vivant en France ne peut demander qu'on lui applique le DI des personnes. De la même façon, si un Français est jugé en Grande-Bretagne pour un délit, il se verra soumis à la procédure britannique en usage et ne peut réclamer qu'on lui applique la procédure française. La raison de cette situation est que le droit étatique s'exerce sur un territoire délimité par des frontières, dans le cadre étatique : un Etat n'est souverain que si sa souveraineté s'exerce pleinement sur le territoire national. C'est une des racines du conflit entre l'Eglise et l'Etat, en Angleterre comme en France. L'Eglise romaine prétendait exercer sa souveraineté à côté de l'Etat national sur le même territoire ; par la même, elle empiétait sur la souveraineté de l'Etat. A l'inverse, le DI ne prétend pas exercer une compétence territoriale, mais seulement sur les personnes qui relèvent de l'islâm. Dès lors qu'une personne a adhéré volontairement au crédo islamique, elle devient un sujet du DI. Dans les pays majoritairement musulmans, où le DI est partiellement ou totalement intégré dans la législation des Etats nationaux, il n'y a pas de conflits majeurs ; dans les pays qui ne sont pas majoritairement musulmans, plusieurs solutions sont possibles. Ou bien on adopte la solution communautaire, comme c'est le cas du Nigéria ou du Soudan. Ou bien on adopte la solution de la souveraineté étatique, comme c'est le cas des Etats ouest-européens. Cette situation fait qu'un musulman sunnite résidant, même de manière passagère, dans un pays musulman où la législation est plus rigoureuse qua dans son pays d'origine, ne peut se prévaloir avec succès de son origine nationale. Par exemple, si un Européen, en principe donc chrétien, se rendait dans un pays musulman et s'il commettait un délit sexuel avec une chrétienne, il ne relevait pas généralement de la justice islamique, mais de la justice de sa communauté. Le fait qu'il soit étranger ne change rien à l'affaire, car ce qui compte, c'est son appartenance confessionnelle. Aujourd'hui, les choses sont un peu différentes car très souvent dans les pays musulmans la loi en vigueur est un mélange des deux registres : droit étatique et DI. Cela fait que le DI, qui a été incorporé souvent dans le droit étatique, peut être appliqué à un non musulman, national ou non.

Il faut reconnaître qu'un autre clivage important a freiné dans le passé l'universalisation du droit : c'est la dichotomie libre-esclave. Il ressort très clairement que le droit, notamment dans le domaine criminel, qui s'applique aux libres ne s'applique pas automatiquement aux esclaves. L'exemple le plus frappant de ce point de vue est le délit d'adultère ou de sexualité pré-nuptiale : dans chacun de ces cas, l'esclave, homme ou femme, n'est tenu qu'à la moitié de la peine du libre. Il ne s'agit pas d'une plus grande clémence pour les hommes de 
condition servile, mais de la prise en considération du fait que l'esclave est le bien de quelqu'un ; si l'on appliquait le droit pénal dans toute sa rigueur, on pourrait faire du tort sur le plan patrimonial au maitre et être tenu pour cette raison de lui restituer une compensation. Cela voudrait dire dans ce cas que la punition de l'esclave coûterait chère à la société et au Trésor public, bayt almâl.

Il n'y a pas, est-il besoin de le souligner, d'effet de la différence des sexes sur le droit positif toutes choses étant égales par ailleurs : le même droit s'applique aux hommes et aux femmes, notamment dans ce que nous appelons aujourd'hui " le civil ». Il y a un domaine en particulier, où la différence des sexes joue un rôle majeur : c'est le pénal. En effet, les femmes ne peuvent intervenir dans la solidarité ('aqila) qui lie entre eux les membres d'un groupe de parenté. Si l'on se place d'un strict point de vue économique (ce qui serait cependant anachronique), cela protège le patrimoine des femmes. Pour payer le prix du sang, si l'un des membres de la parenté s'est rendu coupable d'homicide, seuls les hommes sont sollicités. Dans un autre domaine, la règle qui s'applique aux femmes est différente de celle qui s'applique aux hommes, comme dans le droit successoral du reste : il s'agit du talion. Le prix de la vie d'une femme est inférieur à celui d'un homme, toutes choses étant égales par ailleurs, comme le prix de la vie d'un enfant est inférieur à celui d'un adulte, ou le prix de la vie d'un esclave par rapport à celui d'un libre, ou encore le prix d'un tributaire à celui d'un musulman. Ici il s'agit moins d'universalité que d'asymétrie. ${ }^{7}$

L'islâm est une religion de salut individuel; on ne peut être sauvé en tant que membre d'un groupe, même si cette idée affleure parfois dans l'islâm primitif, comme en témoignent quelques traditions prophétiques qui font état du pouvoir d'intercession de Muhammad en faveur des membres de sa communauté coupables de fautes graves. Ce privilège a pu paraittre arbitraire ou exorbitant aux yeux de certains théologiens rationalistes : si Dieu peut pardonner à un pécheur parce que formellement il était musulman, pourquoi Dieu ne pardonnerait-il pas aux pécheurs appartenant aux autres communautés religieuses ? C'est pour cela que la formulation d'un droit propre à l'islâm est en harmonie avec une théologie rationnelle. On peut même voir dans la formulation

7 L'asymétrie en raison du genre est moins systématique que celle en raison de la condition juridique. d'un tel droit une forme de « rationalisation » de l'islâm. Le DI fixe ainsi un corpus fini de règles, auxquelles le fidèle doit se conformer.

Cela va des règles de la morale sexuelle (condamnation de la sexualité non hétérosexuelle, de la sexualité pré-nuptiale et extra-matrimoniale, etc.) à la morale économique. On peut donc dire que le DI est religieux dans la mesure où il constitue une voie pour le salut après la mort. S’il veut garantir son salut, le fidèle est tenu d'observer scrupuleusement les prescriptions positives et négatives qui sont formulées par le DI. Ainsi on peut comparer la vie du fidèle à un parcours, au cours duquel il est confronté à des situations lui imposant de faire des choix et à l'issue duquel soit il est sauvé soit il est damné. C'est sans doute pour cette raison que le terme sharî‘a, qui signifie « chemin », s'est imposé pour désigner la loi positive : c'est le chemin qui mène au salut. De ce point de vue la sharî́a est comme une sorte de guide qui lui permet de surmonter les obstacles et difficultés, i.e. de faire toujours le bon choix.

Le DI connait deux principales divisions : droit positif (fur̂̀) et théorie légale (usî̀) d'une part, droit cultuel ('ibâdât) et droit des " échanges sociaux » (mu'âmalât) d'autre part. La première distinction est tardive, la seconde est plus ancienne et concerne plus directement notre propos. Le droit des múâmalât recouvre tout ce que dans le monde occidental contemporain on appelle le droit, à savoir le droit des personnes et de la famille, les successions, le droit commercial, le droit pénal de même que le droit de la procédure. Si l'on met de côté une partie du droit pénal, qui concerne ce que l'on appelle hudûd, i.e. les peines édictées par Dieu, et certains aspects de la procédure (recours au serment, voire au tirage au sort), cette partie du droit islamique ne se distingue pas vraiment de n'importe quel autre droit : il s'agit des obligations qui découlent soit de la parenté, soit du contrat, soit enfin du délit. Toutefois, les budûd est la partie du droit pénal qui met en cause trois sujets : la personne accusée, le plaignant et Dieu. Si, par exemple, un homme en blesse un autre, le juge n'aura à statuer sur un tel délit que si la victime porte plainte ; mais celle-ci, ou son représentant, peut s'arranger avec le coupable sans intervention du juge. Si, par contre, un homme en assassine un autre, ou s'il commet un délit sexuel, même si personne ne dépose plainte et si des témoins informent le juge, le ou les coupables seront jugés et condamnés, car ces délits relèvent des budûdce sont des crimes contre Dieu. 
Le droit cultuel ('ibâdât) met en relation non des humains entre eux mais des humains et la divinité. Les principales matières dans cette partie du droit concernent la pureté rituelle, la prière rituelle, le jeûne, le pèlerinage, l'impôt canonique, le serment, le vœu, l'abattage rituel et les interdits alimentaires. L'unité essentielle de toutes ces matières tient au fait qu'il s'agit des obligations contractées par les fidèles vis-à-vis de Dieu : ils les contractent dès lors qu'ils adhèrent à l'islâm. Cependant, on doit distinguer des actes qui mettent en rapport le fidèle et Dieu (par exemple, la prière ou le jeûne) et des actes qui ne mettent en cause Dieu que troisième partie d'une relation entre deux humains c'est le cas du serment - ou entre des humains et des an animaux -abattage rituel, interdits alimentaires. D'ailleurs le serment intervient dans la procédure, puisqu'il en constitue un élément important. C'est sans doute pour cette raison que le terme sharî́, qui signifie " chemin », s'est imposé pour désigner la loi positive : c'est le chemin qui mène au salut. Et c'est de là qu'on en vient à considérer que la sharî́a s'identifie à l'ordre public : s'en prendre à un élément de la sharî‘a c'est s'en prendre à l'ordre public.

Les liens du DI à la religion ne tiennent pas qu'au contenu, ils sont également formels. Nous pouvons en distinguer quatre : $1^{\circ}$ le DI est fondé sur des textes religieux ; $2^{\circ}$ il est l'œuvre d'hommes de religion ; $3^{\circ}$ il est le produit d'un travail exégétique, qui fait appel à des concepts théologico-politiques ; $4^{\circ}$ le champ de la morale ${ }^{8}$ est peu étendu.

Le DI est fondé principalement sur deux textes : le Coran et la Sunna. Cette dernière, qui désigne un " idéal du moi », ${ }^{9}$ repose principalement sur le hadith (au sens large), à savoir l'ensemble du corpus ayant trait à Muhammad et partiellement ses Compagnons les plus éminents. On peut dire que dans le DI, Coran et Sunna constituent un seul ensemble, dans la mesure chacun des textes éclaire l'autre ou est éclairé par lui. C’est pour cette raison que les oulémas tiennent que la loi islamique est d'origine divine et révélée. Le DI comprend une théorie de l'exégèse du Coran, qui a trait principalement

8 On entend ici par morale les actes qui, quand ils ne sont pas conformes à la morale admise, ne sont pas sanctionnés par les pouvoirs en place (par exemple, ne pas tenir sa parole entre amis ou mentir à ses enfants, etc.).

9 Voir J. Laplanche et J.B. Pontalis, Vocabulaire de psychanalyse, PUF, 1981 [1967], 184-186. Selon le propos des auteurs, il « constitue un modèle auquel le sujet cherche à se conformer » (p. 184). mais pas seulement au problème des contradictions entre versets. Cette même théorie précise le statut des textes de la Sunna au regard du Coran. En vérité celle-ci a un rôle sans doute bien plus déterminant puisqu'on lui attribue la fonction insigne de rendre manifestes (bayân) les sens que recouvrent les versets coraniques. Ainsi l'opposition chrétienne entre la lettre et l'esprit doit être évitée absolument : il est par conséquent faux de parler comme on le fait parfois de « littéralisme » à propos des juristes musulmans. C'est vrai que le travail exégétique s'enracine, dans tous les cas, dans la Lettre, mais en recourant à la Tradition et aux usages linguistiques. Prenons un exemple : que signifie le mot nikâh dans le verset 4, 22 ? Selon des juristes, il désigne le mariage, même non consommé, selon d'autres, il désigne toute union sexuelle, y compris avec une esclave ou même avec une étrangère. Même les mystiques sont littéralistes en islâm! C'est le cas par exemple d'Ibn 'Arabî (voir à ce sujet M. Chodkiewicz).

Si les textes révélés constituent la base du DI, celuici est tout de même l'œuvre avant tout des juristes. Car si les textes de la Sunna, en particulier, traitent de nombreux cas, les nouveaux cas ne peuvent être traités que par les spécialistes de ces matières, i.e. les juristes. C'est pour cela qu'il n'est faux de soutenir qu'au cœur du DI, il y a la doctrine formulée par les juristes dans leur enseignement ou dans leurs écrits. C'est là que l'on peut cerner une des différences majeures avec la tradition juridique occidentale. Dans la tradition occidentale, le droit émane de l'Etat, les différents organes de l'Etat se chargeant de le mettre en application ou de le faire respecter (Administration, Police, Justice). Dans le cadre islamique, le droit émane des juristes, qui sont les détenteurs de l'autorité légitime et les gardiens de la sharî‘a. Ils ne sont pas nommés par les titulaires du pouvoir (califes, sultan, émirs) mais sont cooptés par leurs pairs. Ce ne sont pas eux qui dépendent des hommes de pouvoir mais ces derniers qui dépendent d'eux : un émir, qui s'empare du pouvoir par la ruse, l'intrigue ou la violence, a besoin de l'assentiment des oulémas pour voir son pouvoir sinon légitimé du moins accepté par les populations. Dans certaines situations de prise du pouvoir, les oulémas fuient et entrent dans la clandestinité pour ne pas adouber $\left(b_{a y}{ }^{6} a\right)$ par la force les nouveaux potentats. C'est pour cette raison également que les potentats, malgré leur brutalité et souvent leur irréligion, se gardent la plupart du temps de s'en prendre aux oulémas. Pour juger et condamner 
le grand soufi al-Hallâj, il a fallu réunir un tribunal constitué par des oulémas, de simples juges n'auraient pas eu de légitimité. Ce sont également ses pairs qui ont jugé Ibn Taymiyya. ${ }^{10}$ On doit distinguer le juriste et le juge : ce dernier n'est qu'un exécutant, quand il n'est pas lui-même un juriste mujtahid, i.e. capable de traiter les nouveaux cas. Nommé par les détenteurs du pouvoir, qui sont en place en vertu de la force (le pouvoir s'obtient grâce au recours à la force, soit par la conquête, soit par une révolution de palais), le juge doit appliquer en principe un droit élaboré par les juristes. A une époque très ancienne, les Califes ne nommaient pas juges des personnalités compétentes en matière légale ; il est arrivé que des juges soient totalement ignorants dans ce domaine. Les Abbâsides sont les premiers à avoir pris l'habitude de nommer des juristes, parmi lesquels beaucoup d'élèves d'Abû Hanîfa, qui, lui, selon une tradition, aurait refusé l'office de juge. ${ }^{11}$ Il n'y avait pas alors d'écoles personnelles (identifiées par un ancêtre éponyme) et les juristes disposaient d'une très grande liberté d'appréciation. Beaucoup d'entre eux étaient des adversaires d'Abû Hanîfa. Après l'avènement des différentes écoles, les Califes ont pris l'habitude de recruter les juges, selon leur bon gré, là où ils voulaient. Beaucoup plus tard, on fixa la doctrine qui veut que l'on doit nommer dans les grandes métropoles un juge relevant de chacune des écoles reconnues (en principe 4). Plus tard, sous les Ottomans, on étendit ce système aux muftis. La figure du juriste depuis tendit à se confondre avec celle du mufti, seul juriste apte à traiter les cas nouveaux.

\section{Droit et Justice.}

On a l'habitude d'associer droit et justice. Ce n'est pas faux mais n'est exact qu'en partie. Le contrat de droit privé crée du droit sans qu'il y ait intervention du juge ; ce dernier n'intervient qu'en cas de litige, ou de refus d'une partie de remplir ses obligations (par exemple, j'achète une voiture à une société, qui refuse de me la livrer, ou qui me livre une voiture en mauvais

10 D'où le caractère exceptionnel de l'exécution par Nasser en 1966 de Sayyid Qutb un membre influent des Frères musulmans.

11 Mais la tradition est sans doute apocryphe, car il s'agit d'une croyance bien établie parmi les ahl al-hadith, selon laquelle, un docteur de la Loi doit refuser d'occuper la fonction de juge. Il existe à ce sujet plusieurs anecdotes. Abû Haniffa n'appartenait pas aux ahl al-hadith : pouvait-il dès lors adhérer à leur «idéologie »? état, etc.). Mais dans la mesure où il y a dans la vie une place pour le malentendu, le quiproquo, la divergence d'interprétation, des conflits, etc., très souvent c'est au juge qu'il revient de statuer. C'est pour cela que l'on peut avoir tendance à associer droit et justice. Même si le juge constitue une figure importance du monde juridique occidental (on parle même ces dernières années de " judiciarisation »), il n'est au mieux qu'un interprète de la Loi, au pire un exécutant ; mais luimême n'est pas à l'origine de la Loi. En France, et de manière générale dans ce que l'on a l'habitude d'appeler Occident, la Loi est l'œuvre de celui qu'on appelle le Législateur même si l'on a pris l'habitude, en France comme aux EU, de désigner une loi par celui qui en pris l'initiative ou qui a déposé devant le Parlement le projet. Il s'agit bien évidemment d'une fiction institutionnelle. Le Législateur est une manière métaphorique de désigner le Parlement. Pour autant, vous le savez, tant qu'une loi n'est pas édictée par le pouvoir exécutif, elle n'a pas de réalité. Le pouvoir législatif, comme on dit habituellement, est ainsi distingué du pouvoir exécutif pour des raisons d'équilibre institutionnel, mais cette distinction ne doit pas cacher que les deux pouvoirs, de même d'ailleurs que le troisième pouvoir - celui des juges - ne sont que des organes de l'Etat. C'est pour cela que dans l'Etat moderne, seul l'Etat est le législateur réel. D'ailleurs, la distinction des pouvoirs cache mal que la plupart des lois sont prises à l'initiative du pouvoir exécutif qui s'accorde avec le pouvoir législatif, les deux pouvoirs étant l'expression, dans les démocraties représentatives, du suffrage universel. Dans le cas français, cela est patent depuis l'émergence de la monarchie au Moyen Age : jusqu'à 1789, les lois étaient édictées par le monarque, après la Révolution et sauf période d'interrègne comme l'Empire, les lois sont votées par le parlement et édictées pour le détenteur du pouvoir exécutif (monarque, président). Dans ce cas, les juges ont un rôle bien modeste : ils appliquent les lois que d'autres élaborent et décident. Le seul domaine où ils interviennent c'est celui de l'interprétation et qui peut donner matière à jurisprudence.

Dans le système de DI, les juges doivent être distingués des juristes. Le DI est un droit de juristes : c'est sans doute le trait distinctif essentiel de ce droit, parmi les systèmes juridiques dans le monde. Cela l'oppose notamment à la tradition occidentale, dans laquelle l'Etat joue le rôle déterminant : en effet dans le monde occidental moderne, c'est surtout l'Etat 
qui est à l'origine du droit, en particulier des lois, qui définissent l'ordre juridique dans lequel chacun évolue. Les différences entre les ordres juridiques étatiques ne remettent pas en cause cette unité fondamentale. De ce point de vue, le DI ressemble à ce qu'en France on appelle « la doctrine », qui est l'œuvre des professeurs de droit : cependant alors que la doctrine n'a point d'autorité, en D I, la doctrine prime sur l'activité des juges, voire même elle s'identifie au droit proprement dit. Le DI est de ce point de vue proche, en partie, du droit romain, qui était lui aussi fondé sur l'activité de juristes privés. Cependant dans le système romain, les doctrines des juristes avaient besoin du concours de l'Empereur pour être élevées en position d'autorité ; les juristes musulmans exercent un pouvoir en vertu même de leur position dans la société. Un autre trait important les distingue des juristes romains : ils sont en même temps des hommes de religion. Ce dernier trait les rapproche des rabbins. D'ailleurs le système dont le DI est sans doute le plus proche est le droit rabbinique.

Le fait que le DI soit un droit de juristes a un certain nombre de conséquences importantes, qui lui confèrent sa singularité. Dans la mesure où il s'agit d'un droit de juristes, il est automatiquement un droit savant, qui se transmet dans le cadre d'un enseignement. Ce qui suppose des lieux pour l'enseigner, une organisation ainsi qu'un financement. Dès la constitution du DI, vont apparaittre les madrasa dans les grandes régions du monde islamique, financées soit par des mécènes, qui sont souvent les hommes de pouvoir du moment, qui veulent s'assurer le soutien ou la neutralité bienveillante des hommes de religion, ainsi qu'une garantie pour l'audelà, soit par des awqā $f$, «fondations pieuses ». Pendant longtemps, les oulémas devaient exercer une activité économique propre afin de subvenir à leurs besoins et à celle de leur famille : cela apparaît notamment dans les noms de relation dont on les affublés parfois.

Une autre conséquence importante est que le DI ne peut évoluer ou progresser que grâce à l'intervention directe des juristes. Les hommes de pouvoir n'y peuvent absolument rien. C'est la fameuse question de l'ijtibâd et du taqiid.

Une troisième conséquence est que le DI recouvre diverses interprétations. C'est pour cette raison que la sharîa prend des visages diversifiés. Elle est une mais a plusieurs versions. C'est la question de ce qui a été théorisé comme ikhtilâf, qui est une des branches les plus importantes du fiqh. On s'est efforcé tout de même de poser des limites à la liberté de l'interprétation en mettant en place quatre écoles juridiques au sein du sunnisme ainsi que grâce à la théorie légale. Malgré tout, cette limitation n'a pu entièrement supprimer toute possibilité de contestation de l'ordre juridique établi : n’importe quel juriste, pourvu que sa compétence soit reconnue par ses pairs, peut formuler une doctrine dissidente. C'était le cas par exemple d'Ibn Taymiyya. Toutes proportions gardées, c'est le cas aujourd'hui d’Usâma b. Ladin.

\section{LES SOURCES DU DI.}

Les sources matérielles du DI sont le Coran et la Tradition. Le DI ignore le concept de droit naturel, mais le recours au raisonnement occupe une place plus importante qu'on ne le croit. De nombreux concepts qui jouent un rôle dans la théorie légale sont du type rationnel :

- le raisonnement par analogie (qiyâs), selon lequel pour traiter un cas nouveau on doit le rapporter à un ancien cas connu, en mettant l'accent sur la ratio legis, 'illa, d'une règle, c'est-à-dire la raison d'être d'une loi (on peut citer l'exemple des boissons alcoolisées inconnues du Coran mais qui sont prohibées);

- le principe de continuité (istiṣhâhb), selon lequel tant qu'un usage ou une règle n'entre pas en contradiction avec la DI, il ne doit pas être remis en cause alors même qu'il est étranger au DI ;

- le principe de la maslaha, qui doit être entendu à la fois comme l'intérêt légitime du sujet de droit (par exemple, il est de l'intérêt d'un individu de défendre sa vie) et l'intérêt général, sachant que les intérêts de l'individu et de la collectivité sont complémentaires ;

- le principe de l'istihsân selon lequel entre deux solutions, on doit préférer celle qui est la plus avantageuse (on peut donner l'exemple de l'enfant dont la paternité est revendiquée par un libre et un esclave, ou un musulman et un non musulman). 


\section{Conclusion.}

La tradition juridique islamique est d'une très grande originalité. C'est pour cela qu'il est vain, pour ne pas dire dangereux, de la rapporter à la tradition occidentale moderne et de la considérer à travers les catégories de celles-ci prises comme universelles. Nous avons essayé d'en esquisser certains aspects caractéristiques, mais il faudrait bien sûr plus de développements pour tenter de la cerner plus systématiquement. Ce qui est sans doute au cœur de cette tradition, c'est l'idée d'interprétation du juriste-théologien. 
Para publicar na Revista de Direito Internacional, acesse o endereço eletrônico www.rdi.uniceub.br ou www.brazilianjournal.org.

Observe as normas de publicação, para facilitar e agilizar o trabalho de edição. 